\title{
Monoclonal antibodies as probes for fungal wall structure during morphogenesis
}

\author{
Margaret Marshall, ${ }^{1}$ Keith Gull ${ }^{2}$ and Peter Jeffries ${ }^{1}$ \\ Author for correspondence: Peter Jeffries. Tel: +44 1227823478. Fax: +44 1227787465. \\ e-mail: P.Jeffries@ukc.ac.uk
}

1 Research School of
Biosciences, The University
of Kent, Canterbury, Kent
CT2 7NJ, UK
2 School of Biological
Sciences, 2.205 Stopford
Building, University of
Manchester, Oxford Road,
Manchester M13 9PT, UK Manchester M13 gPT, UK

\begin{abstract}
Three monoclonal antibodies (mAbs), S4D1, S3B3 and S1E5, were produced from hybridoma cell lines raised from mice immunized with hyphal walls of Neurospora crassa and one (Pax-1) from mice immunized with hyphal walls of Paxillus involutus. In immunofluorescence studies, the three $\boldsymbol{N}$. crassa mAbs recognized epitopes with different patterns of distribution at the hyphal surface of $N$. crassa. S4D1 recognized an epitope which was present on the surface of both conidia and hyphae; S3B3 recognized an epitope seen only at the ends of conidia or in the septal region of hyphae and conidial chains; and S1E5 recognized an epitope present on the surface of hyphae, but not on mature conidia. mAb Pax-1 reacted with hyphal wall fragments of Pax. involutus and with $N$. crassa conidia in a similar way to S3B3. S4D1 reacted with an epitope found in 1,3-x-glucan preparations from hyphal walls of different fungi. The surface distribution of this epitope varied: it was found on the surface of both conidia and hyphae of $N$. crassa and Aspergillus nidulans, on the basidiospore surface only of Amanita muscaria, and on the hyphae but not the conidia of Penicillium chrysogenum. Immunogold studies revealed that the epitope was present throughout the wall of conidia and hyphae of $N$. crassa. mAbs S3B3, S1E5 and Pax-1 also reacted with other fungi: for example Pax-1 cross-reacted with all fungi tested except for a member of the Zygomycota. Immunogold studies revealed that epitopes of these three mAbs were present within the inner layers of the walls of conidia and hyphae of N. crassa.
\end{abstract}

Keywords: monoclonal antibodies, immunofluorescence, hyphal structure, Neurospora crassa, Paxillus involutus

\section{INTRODUCTION}

The use of mAbs as specific probes for the presence of certain fungi has received considerable attention in mycology, particularly for rapid diagnosis of plant disease (Miller \& Martin, 1988; Schots et al., 1994). There is also, however, the potential to use mAbs as probes to study the differential distribution of specific wall components within different fungal structures, or to monitor very local changes of wall architecture and biochemistry during hyphal and spore morphogenesis. The fungal wall is responsible for the characteristic shape of fungal structures, and information about the wall components and their distribution is important in our understanding of their role in cell integrity and

Abbreviations: IF, immunofluorescence; PC, phase-contrast. morphogenesis. Fungal walls comprise of numerous insoluble and soluble components, many of which are immunogenic, and the latter in particular have been chosen for the development of species-specific probes in diagnosis. Wall-bound antigens, however, lend themselves readily to adaptations of microscopic quantification techniques, and the durability of the walls themselves minimizes the loss of antigen during extraction procedures. Such antigens, however, may not be useful as specific tools to distinguish individual species as many epitopes within hyphal walls are common to a range of fungi and some may be immunodominant. The use of purified, insoluble hyphal wall fragments as immunogens has been less frequent than the use of soluble immunogens, but several studies (e.g. Breuil et al., 1992; Thornton et al., 1993) have been successful especially in medical mycology (Ste-Marie et al., 1990). 
The molecular architecture of hyphal walls remains poorly documented, and most information has been derived from electron microscopy of sections of intact walls or from shadow-cast residues from drastic chemical treatments (Hunsley \& Burnett, 1970). Immunofluorescent probes have been less frequently used, although Hunsley \& Kay (1976) pioneered the use of polyclonal antisera to correlate immunological observations with previous ultrastructural studies. More recently, immunoconjugates such as chitin-specific lectins (Pain et al., 1994a), hyphal-wall-degrading enzymes (Benhamou et al., 1990), and polyclonal and monoclonal antibodies raised against specific glucans (Wycoff \& Ayers, 1990; Gianinazzi-Pearson et al., 1994) have been used to localize these epitopes within specific regions of ultrathin sections of fungal walls. The importance of the need for ultrastructural localization of antigens, as well as immunofluorescence (IF) labelling methods, was emphasized by Cole et al. (1991) who demonstrated the differential localization of a specific epitope within a range of structures formed by Penicillium islandicum.

In the past there have been problems in devising methods to stick insoluble hyphal fragments to microtitre plates for screening of hybridoma supernatants, but the use of ELISA screening assays in microtitre plates coated with hyphal fragments has now been developed (Banks \& Cox, 1992; Breuil et al., 1992) which enables these targets to be used in the screening procedures. The work described here was initiated using Neurospora crassa, a fungus with a well-characterized wall biochemistry, to develop procedures for the isolation of $\mathrm{mAbs}$ specific for insoluble antigens, and to determine their usefulness in investigations of wall architecture. It was extended to include Paxillus involutus, an ectomycorrhizal fungus, as a subsequent aim of the work is to use these probes to study wall morphogenesis within the developing plantfungus interface during initiation of this ecologically important symbiosis.

\section{METHODS}

Fungi. N. crassa IMI 142819 was used to raise antibodies and was maintained on $N$. crassa minimal medium (NCMM; Difco) in Petri dishes. Stock cultures were stored at $4{ }^{\circ} \mathrm{C}$ on media slopes in Universal bottles. Neurospora crassa IMI 53240, Neurospora sitophila IMI 63920, and Neurospora tetrasperma IMI 34454 were also grown in the same way and used to screen hybridoma supernatants. Large quantities of conidia from Neurospora spp. were obtained from cultures grown on NCMM slopes in 250 and $500 \mathrm{ml}$ medical flat bottles. Pax. involutus ITE16 was also used to raise antibodies and was obtained from the Institute of Terrestrial Ecology (ITE), Penicuik, UK, and grown on Modified Melin-Norkrans medium (MMN; Marx, 1969) as above. Pax. involutus ITE32 and Pax. involutus ITE35 were also obtained from the ITE and grown on this medium and used to screen hybridoma supernatants. Other fungi used for screening of hybridoma supernatants were grown on malt extract agar (Oxoid) at $25^{\circ} \mathrm{C}$ and included Aspergillus nidulans IMI 16040, Colletotrichum gloeosporioides UKC24, Geotrichum candidum UKC50, Mucor hiemalis IMI 21216, Penicillium chrysogenum IMI 37767, Podospora paucieta CBS29356, Schizophyllum commune UKC17, Sordaria fimicola IMI 105391. Basidiomata of Amanita muscaria along with another 20 species of Basidiomycota were collected from the campus of the University of Kent and identified, whereas basidiomata of Agaricus bisporus were purchased from a local shop. In both cases, spore and hyphal samples were used in screening studies.

Germination of $\boldsymbol{N}$. crassa conidia in batch culture. Conidia were harvested from $20-30$-d-old slope cultures by washing the surface of the culture with sterile $0.1 \%(\mathrm{v} / \mathrm{v})$ Tween 80 solution. The suspension of conidia was filtered through sterile glass wool and the number of conidia per $\mathrm{ml}$ in the resulting suspension counted. Erlenmeyer flasks $(250 \mathrm{ml})$ containing $25 \mathrm{ml}$ Neurospora germination medium (Chang $\&$ Trevithick, 1972) or Neurospora culture broth (NCB; $5 \mathrm{~g}$ Difco yeast extract $\mathrm{l}^{-1}, 5 \mathrm{~g}$ Difco Proteose peptone $\mathrm{l}^{-1}, 40 \mathrm{~g}$ maltose $\mathrm{l}^{-1}$ ) were inoculated with $1 \times 10^{6}$ conidia $\mathrm{ml}^{-1}$. Duplicate flasks were prepared for each time point (up to $12 \mathrm{~h}$ ). The cultures were incubated at $30^{\circ} \mathrm{C}$ and agitated at 200 r.p.m. At hourly intervals, duplicate flasks were removed from the shaker and $25 \mathrm{ml}$ freshly prepared formaldehyde solution $(7 \cdot 4 \%, \mathrm{w} / \mathrm{v})$ was added, giving a final formaldehyde concentration of $3.7 \%(w / v)$. After $30 \mathrm{~min}$ fixation, germinated conidia were washed and resuspended in sterile distilled water.

Mini-cultures of $\boldsymbol{N}$. crassa. Mini-cultures of $N$. crassa were grown to obtain intact conidiophores for IF studies of epitope distribution. Aliquots $(100 \mu \mathrm{l})$ of NCMM were placed into the wells of a 96-well microtitre plate. Each well was seeded with approximately $100 \mathrm{~N}$. crassa conidia and incubated at $30^{\circ} \mathrm{C}$, in the light, for $3 \mathrm{~d}$ until the fungus formed a thin mycelial mat on the surface of the medium. The medium was then removed by aspiration and the culture fixed for $30 \mathrm{~min}$ by addition of $3.7 \%(\mathrm{w} / \mathrm{v})$ freshly prepared formaldehyde solution.

Culture of fungi for hyphal wall isolation. For the preparation of hyphal walls of $N$. crassa IMI 142819, a 101 carboy containing 91 NCMM minus sucrose was autoclaved for $40 \mathrm{~min}$ at $121^{\circ} \mathrm{C}, 15$ p.s.i. Following sterilization, $200 \mathrm{~g}$ sucrose in $750 \mathrm{ml}$ distilled water was added and the final medium allowed to equilibrate to $30^{\circ} \mathrm{C}$. A conidial suspension of $N$. crassa was prepared from a 20-30-d-old culture maintained on an NCMM agar slope in a $500 \mathrm{ml}$ medical flat bottle by harvesting conidia in sterile $0.1 \%$ Tween 80 and filtering it through sterile glass wool. The volume of the suspension was adjusted so that the final concentration of conidia in the carboy would be $1 \times 10^{5}$ conidia $\mathrm{ml}^{-1}$. After inoculation the carboy was incubated at $30^{\circ} \mathrm{C}$ for $3 \mathrm{~d}$ on a magnetic stirrer and was connected to an air supply via an air inlet pipe. The air passed through a $0.2 \mu \mathrm{m}$ filter before being dispersed throughout the culture by a glass sparger. An air outlet pipe fitted with a $0 \cdot 2 \mu \mathrm{m}$ filter allowed release of gas from the culture vessel. The medium was agitated by means of a large stirrer bar. For preparation of hyphae of Pax. involutus, five 21 Erlenmeyer flasks containing $200 \mathrm{ml} \mathrm{MMN}$ broth were inoculated with Pax. involutus ITE16 using a plug from a Petri dish culture. The flasks were incubated statically at $20^{\circ} \mathrm{C}$, in the dark. The mycelium was collected when the mycelial mat covered the surface of the medium, usually after 1-2 months.

Isolation of hyphal walls. Hyphal walls of N. crassa and Pax. involutus were isolated for use in immunization, ELISA and IF protocols as follows. Mycelium was removed from culture media by filtering through four layers of muslin. It was then split into aliquots of approximately $25 \mathrm{~g}$ (wet weight). Aliquots not used immediately were stored at $-80^{\circ} \mathrm{C}$. All other aliquots were subjected to the following breakage and cleaning procedure: mycelium was passed three times through 
a French pressure cell, at a pressure of 14087 tonnes $\mathrm{m}^{-2}$. The resulting mixture of wall fragments and cytoplasm was then spun at 4500 r.p.m. in an MSE Centaur bench-top centrifuge $(2250 \mathrm{~g})$ for $5 \mathrm{~min}$. The supernatant was resuspended in sterile distilled water, before being sonicated in an MSE PG100 sonicator $(16 \mu \mathrm{m}$ amplitude peak-to-peak, $5 \mathrm{~min}$ in total, in $10 \mathrm{~s}$ bursts with $10 \mathrm{~s}$ intervals). The suspension was then recentrifuged and the sonication repeated until cytoplasmic contamination [checked using phase-contrast (PC) microscopy and by a negative result to the orcinol test for RNA] was removed. The isolated walls were then lyophilized for storage.

Fractionation of hyphal walls of $\boldsymbol{N}$. crassa. Isolated $N$. crassa cell walls were fractionated into four crude cell wall fractions according to the method of Mahadevan \& Tatum (1965). This involves sequential acid and alkali hydrolysis and the four fractions comprise galactosamine-peptide-glucan (Fraction I), monosaccharide sugars (Fraction II), glucan (Fraction III), and chitin (Fraction IV), respectively. The fractions obtained were lyophilized for storage. Samples of pure chitin, $\mathrm{N}$-acetylglucosamine and galactosamine (Sigma), 1,3- $\alpha$-glucan from Aspergillus nidulans (from Professor B. J. M. Zonneveld, Leiden, The Netherlands), and R- and S-glucan from Schiz. commune (from Professor J. G. H. Wessels, Haren, The Netherlands) were similarly lyophilized and prepared for ELISA for comparative binding assays.

Development of an immunization schedule for mAb production. To determine the appropriate level of immunogen to be used, two sets of three $\mathrm{BALB} / \mathrm{c}$ mice were given intraperitoneal injections with 1 and $0.5 \mathrm{mg}$ (freeze-dried weight) of hyphal walls, respectively. These amounts were chosen because larger amounts of material are known sometimes to be fatal. Prior to injection the walls were suspended in sterile distilled water and sonicated to produce a homogeneous suspension. The timing of the injection was such that each mouse was give one injection of the appropriate dose on days $1,7,14,28$ and 49, five injections in total. All of the mice were bled from the tail vein on day 59, $10 \mathrm{~d}$ after the fifth injection. Plasma from each mouse was tested for its reaction with hyphal walls of $N$. crassa using ELISA. The $1 \mathrm{mg}$ dose produced a much higher end-point titre $(>1: 18000)$ than the $0.5 \mathrm{mg}$ dose and injections of $1 \mathrm{mg}$ were subsequently used for all injections, with approximately $14 \mathrm{~d}$ between each. A booster injection ( $1 \mathrm{mg}$ ) was given $4 \mathrm{~d}$ before a mouse was killed for fusion.

Production of mAbs. Spleen cells were fused with $\mathrm{Sp} 2.0$ myeloma cells at a ratio of 5:1 following standard fusion protocols involving the addition of $75 \mathrm{mM}$ polyethylene glycol (Marshall, 1990). Hybridoma supernatants were screened initially using a non-competitive ELISA with hyphal walls bound directly to the plate. Hybridomas were considered positive if their optical density was greater than 0.88 for $N$. crassa as the antigen and 0.78 if Pax. involutus was the antigen. These values are the mean plus four standard deviations when non-specific mAbs were tested in the ELISA for their reaction with the hyphal walls. The hybridomas with positive supernatants in the ELISA were then tested by IF. Hybridomas whose supernatants showed different fluorescence patterns were cloned. mAbs were typed by immunodiffusion tests against sheep anti-mouse antisera (ICN) raised against the antibody heavy-chain classes $\operatorname{IgG}_{1}, \operatorname{IgG}_{2 \mathrm{a}}, \operatorname{IgG}_{2 \mathrm{~b}}, \operatorname{Ig} A$ and $\operatorname{Ig} M$.

ELISA. Because of initial difficulties in binding hyphal walls of $N$. crassa directly to ELISA plates, an extensive series of comparative experiments was completed (Marshall, 1990) to determine the most appropriate methodology (binding agents, drying times, etc.) and optimum assay conditions (antibody concentrations, etc.) for an indirect non-competitive ELISA. The final protocol selected was determined initially with polyclonal sera (raised using standard protocols in New Zealand White rabbits using hyphal walls as immunogen). Strong and even binding of hyphal fragments to the ELISA plates was accomplished by initially coating wells with poly-Llysine $\left(10 \mu \mathrm{g} \mathrm{ml}^{-1}\right)$, then pipetting $100 \mu \mathrm{l}$ hyphal wall suspension at $100 \mu \mathrm{g}$ (freeze-dried weight) $\mathrm{ml}^{-1}$ in sterile distilled water into wells to give an antigen concentration of $10 \mu \mathrm{g}$ per well. Material was then allowed to dry on to the plate at $37^{\circ} \mathrm{C}$ overnight. BSA $\left(250 \mu 11 \%, \mathrm{w} / \mathrm{v}\right.$, in PBS : $8.0 \mathrm{~g} \mathrm{NaCl} \mathrm{l}^{-1}, 0.2 \mathrm{~g}$ $\mathrm{KCl} \mathrm{l}^{-1}, 2 \cdot 16 \mathrm{~g} \mathrm{Na}_{2} \mathrm{HPO}_{4} .7 \mathrm{H}_{2} \mathrm{O} \mathrm{l}^{-1}, 0 \cdot 2 \mathrm{~g} \mathrm{KH}_{2} \mathrm{PO}_{4} \mathrm{l}^{-1}$; $\mathrm{pH} \mathrm{7 \cdot 0)}$ was added to each well and the plate incubated for $1 \mathrm{~h}$ at $37^{\circ} \mathrm{C}$. The blocker was flicked from the wells and $100 \mu$ of the first antibody was added and the plate incubated for $2 \mathrm{~h}$ at $37^{\circ} \mathrm{C}$. After incubation the antibody solution was flicked from the plate and the wells were washed six times for $5 \mathrm{~min}$ with $300 \mu \mathrm{l}$ PBS containing $0.05 \%(\mathrm{v} / \mathrm{v})$ Tween 20. Second antibody, either anti-rabbit or anti-mouse IgG conjugated to horseradish peroxidase (Sigma) was diluted 1:500 in PBS and $100 \mu \mathrm{l}$ added to each well. The plate was then incubated for $2 \mathrm{~h}$ at $37^{\circ} \mathrm{C}$ and the same washing procedure followed as after the first antibody incubation. 2,2-Azinobis(3-ethylbenz-thiazolinesulphonic acid) was used as the substrate at a concentration of $0.8 \mathrm{mM}$ in $0.1 \mathrm{M}$ citrate buffer.

IF. Polyclonal antisera and hybridoma supernatants were screened using an indirect IF technique. Slides were coated with poly-L-lysine $\left(100 \mu \mathrm{g} \mathrm{ml}^{-1}\right)$, by submersion of the slides in the poly-L-lysine solution for $15 \mathrm{~min}$. Excess poly-L-lysine was removed by a wash in PBS and the slides air-dried. Ten microlitre aliquots of a suspension of test fungal material were placed onto the slides. For screening of hybridoma supernatants this comprised either $10 \mu \mathrm{l}$. crassa germlings, or $10 \mu \mathrm{l}$ Pax. involutus hyphal walls. The $10 \mu \mathrm{l}$ aliquots were airdried on the slides for $30-60 \mathrm{~min}$ at $25^{\circ} \mathrm{C}$. Ten microlitres of the first antibody was then placed onto each sample and the slides incubated at $25^{\circ} \mathrm{C}$ for $1 \mathrm{~h}$ in moist conditions. The slides were then washed three times for 5 min with PBS. Excess PBS was drained from the slides and $10 \mu \mathrm{l}$ of either anti-mouse IgG or anti-rabbit IgG FITC conjugate (Janssen) diluted 1:10 in PBS was placed on each sample before incubation at $25^{\circ} \mathrm{C}$ for $1 \mathrm{~h}$. The washing step $(3 \times \mathrm{PBS}, 5 \mathrm{~min})$ was repeated and the slides drained. A drop $(10 \mu \mathrm{l})$ of Mowiol (Sigma) containing $10 \mu \mathrm{g} \mathrm{ml}^{-1} p$-phenylenediamine was added and samples were observed by epifluorescence using a Zeiss Axioskop microscope with a mercury lamp and a Zeiss standard dichroic filter set no. 10 for detection of transmitted light from FITC. Checks were made during all experiments that the fluorescence was not due to non-specific binding by comparing results with second antibody controls (i.e. preparations in which the first antibody but not the second was omitted) using fungal spores, hyphae and the undiluted culture medium as substrates.

Immunogold electron microscopy. Mini-cultures of $N$. crassa were grown in the wells of microtitre plates, fixed with $3.7 \%$ (w/v) formaldehyde containing $0.6 \%$ glutaraldehyde in PipesEDTA-magnesium (PEM) buffer for 1-2 $h$, and dehydrated and embedded in Lowicryl K4M (Chemische Werke Lowi) using the standard protocol involving infiltration during progressive lowering of the temperature prior to polymerization at $-35^{\circ} \mathrm{C}$ using UV light (Bendayan, 1984). Thin sections $(150-190 \mu \mathrm{m})$ were cut using a Reichert ultratome and mounted on nickel grids for immunogold labelling. Subsequent steps were carried out by placing one drop $(10 \mu \mathrm{l})$ of the appropriate solution onto each grid and incubating them in a bioassay plate at room temperature. Free aldehyde groups were neutralized by three $5 \mathrm{~min}$ washes in $20 \mathrm{mM}$ glycine. The 


\section{Table 1. IF reactions of $m A b s S 4 D 1, S 3 b 3$ and Pax-1 with a taxonomically diverse range of fungi}

+++ , Strong fluorescence; ++ , moderate fluorescence; + , weak fluorescence; - , no reaction. NT, combination was not tested.

\begin{tabular}{|c|c|c|c|}
\hline \multirow[t]{2}{*}{ Fungus } & \multicolumn{3}{|c|}{$\mathrm{mAb}$} \\
\hline & S4D1 & S3B3 & Pax-1 \\
\hline \multicolumn{4}{|l|}{ Ascomycota } \\
\hline \multicolumn{4}{|l|}{ Sordariaceae } \\
\hline Neurospora crassa IMI 142819 & +++ (All surfaces $)$ & $\begin{array}{l}+++ \text { (Some conidial } \\
\text { ends, septa in } \\
\text { conidiophore chains) }\end{array}$ & $\begin{array}{l}+++(\text { Some } \\
\text { conidial ends })\end{array}$ \\
\hline Neurospora crassa IMI 53240 & +++ (All surfaces) & $+++($ As above $)$ & NT \\
\hline Neurospora sitophila & +++ (All surfaces) & +++ (As above $)$ & NT \\
\hline Neurospora tetrasperma & +++ (All surfaces) & +++ (As above $)$ & NT \\
\hline Sordaria fimicola & $\begin{array}{l}++ \text { (Asci, ascospores }) \\
+ \text { (Hyphae })\end{array}$ & - & NT \\
\hline \multicolumn{4}{|l|}{ Chaetomiaceae } \\
\hline Chaetomium globosum & - & NT & nt \\
\hline \multicolumn{4}{|l|}{ Dipodascaceae } \\
\hline Geotrichum candidum & - & - & - \\
\hline \multicolumn{4}{|l|}{ Lasiosphaeriaceae } \\
\hline Podospora paucieta & - & - & NT \\
\hline \multicolumn{4}{|l|}{ Anamorphic fungi } \\
\hline \multirow[t]{2}{*}{ Aspergillus nidulans } & +++ (Hyphae $)$ & & \\
\hline & $++/+$ (Conidia) & - & - \\
\hline Colletotrichum gloeosporioides & $\begin{array}{l}++ \text { (Secondary germ tubes }) \\
- \text { (Conidia, appressoria, } \\
\text { primary germ tubes })\end{array}$ & NT & + (Germ tubes only) \\
\hline Penicillium chrysogenum & $\begin{array}{l}+++ \text { (Hyphae, conidiophores }) \\
-(\text { Phialides, conidia) }\end{array}$ & $\begin{array}{l}- \text { (Hyphae }) \\
+ \text { (Conidial ends })\end{array}$ & + (Conidial ends $)$ \\
\hline \multicolumn{4}{|l|}{ Basidiomycota } \\
\hline \multicolumn{4}{|l|}{ Agaricaceae } \\
\hline Agaricus bisporus & NT & - & +++ \\
\hline \multicolumn{4}{|l|}{ Amanitaceae } \\
\hline Amanita muscaria & $\begin{array}{l}- \text { (Hyphae) } \\
+++ \text { (Basidiospores) }\end{array}$ & NT & NT \\
\hline \multicolumn{4}{|l|}{ Paxillaceae } \\
\hline Paxillus involutus & ++ (Hyphae $)$ & - & +++ \\
\hline \multicolumn{4}{|l|}{ Schizophyllaceae } \\
\hline Schizophyllum commune & $\begin{array}{l}+++ \text { (Skeletal hyphae) } \\
+ \text { (Generative hyphae) }\end{array}$ & - & +++ \\
\hline \multicolumn{4}{|l|}{ Zygomycota } \\
\hline \multicolumn{4}{|l|}{ Mucoraceae } \\
\hline Mucor biemalis & - & - & - \\
\hline
\end{tabular}

grid was then washed once with PBS and incubated with the first antibody for $1 \mathrm{~h}$. The mAb hybridoma supernatants were not diluted. The grid was then washed five times for $5 \mathrm{~min}$ with $1 \%$ BSA/PBS. Anti-mouse IgG whole-molecule $10 \mathrm{~nm}$ diameter gold conjugate (Janssen) was diluted $1: 10$ in $1 \%$ BSA/PBS and placed on the grid for $1 \mathrm{~h}$ before washing twice in $1 \% \mathrm{BSA} / \mathrm{PBS}$ for $5 \mathrm{~min}$, followed by three $5 \mathrm{~min}$ washes in $0 \cdot 1 \%$ BSA/PBS and two 5 min washes in PBS. The bound antibody was fixed to the sections by incubation in $2.5 \%(\mathrm{v} / \mathrm{v})$ glutaraldehyde for $30 \mathrm{~s}$. Sections were then stained using $5 \%$ $(\mathrm{w} / \mathrm{v})$ uranyl acetate in $1 \%(\mathrm{v} / \mathrm{v})$ acetic acid and Reynold's lead citrate. Grids were observed using a Philips EM410 transmission electron microscope. Control preparations in which the first antibody (but not the second) was omitted were always run in parallel to check for non-specific binding.

\section{RESULTS}

\section{Production of mAbs}

After initial screening by ELISA of immunized mouse antisera and resulting hybridoma supernatants, hybridomas deemed positive by comparison to known polyclonal antisera were assayed by IF. Of 3360 super- 

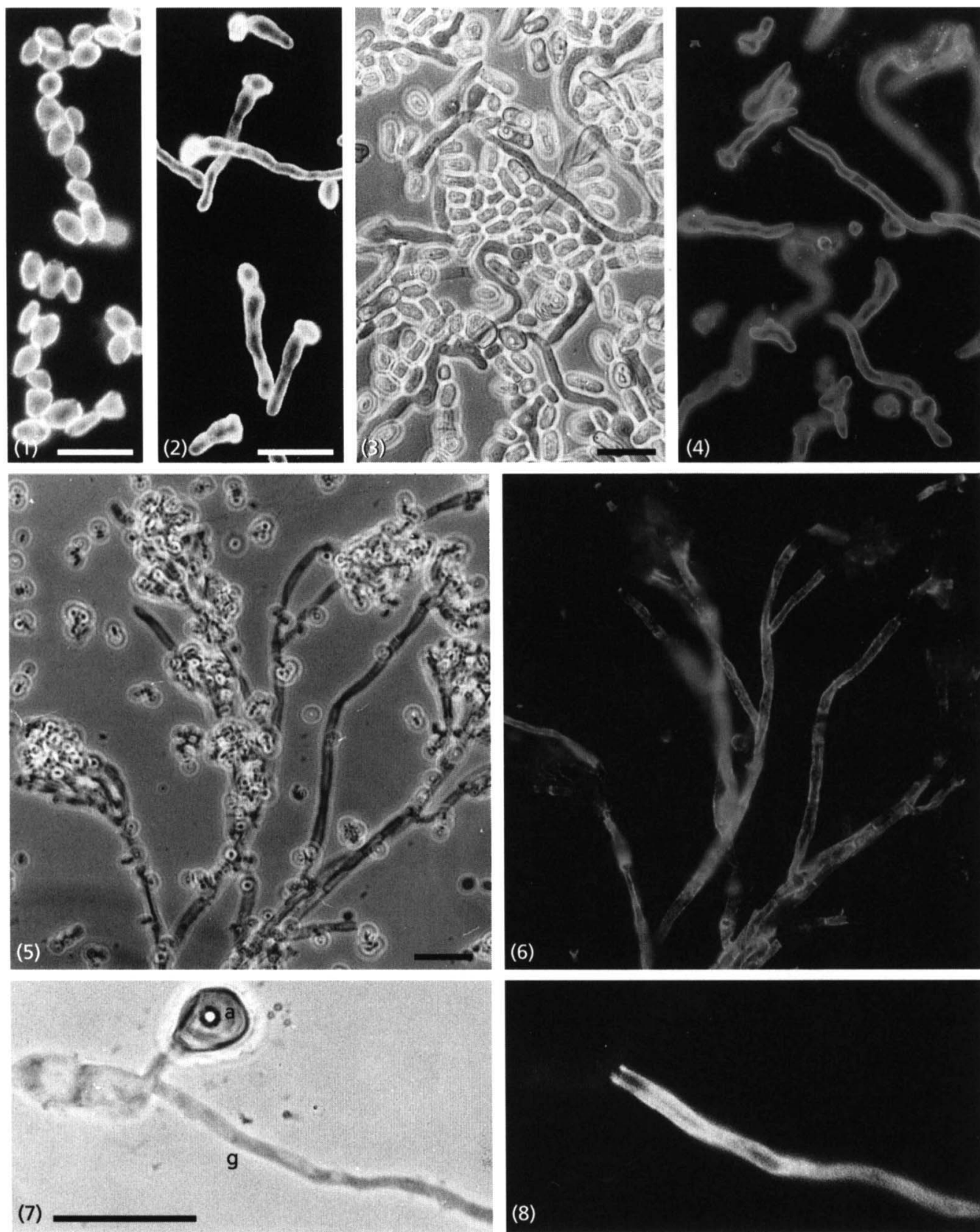

Figs 1-8. Fig. 1. IF micrograph of ungerminated conidia of $N$. crassa treated with mAb S4D1 showing even distribution of epitope over conidial surface. Bar, $15 \mu \mathrm{m}$. Fig. 2. IF micrograph of germlings of $N$. crassa treated with mAb S4D1 showing even distribution of epitope over surface of germ tubes and conidia. Bar, $15 \mu \mathrm{m}$. Fig. 3. PC micrograph of a mixture of germlings of $N$. crassa and arthroconidia of $G$. candidum treated with mAb S4D1. Bar, 15 $\mu \mathrm{m}$. Fig. 4. IF image corresponding to Fig. 3 showing selective binding of mAb to $N$. crassa. Fig. 5. PC micrograph of a conidiophore of Pen. chrysogenum treated with mAb S4D1. Bar, $15 \mu \mathrm{m}$. Fig. 6. If image corresponding to Fig. 5 showing localization of epitope on conidiophore but not on conidia or phialides. Fig. 7. PC micrograph of a germinating conidium, secondary germ tube (g) and appressorium (a) of Col. gloeosporioides treated with mAb S4D1. Bar, 15 $\mu \mathrm{m}$. Fig. 8. IF image corresponding to Fig. 7 showing even distribution of labelling over surface of secondary germ tubes only.

natants from hybridoma cell lines obtained from mice injected with $N$. crassa, 286 tested positive by ELISA and 102 of these recognized hyphal fragments of $N$. crassa as determined by IF. The patterns of fluorescence fell into four major groups: those which reacted with: (i) conidial ends and septa, (ii) hyphae and conidia, (iii) hyphae only, and (iv) spores only. Hybridoma cell lines which fell into the first three categories were successfully cloned and were represented by mAbs S3B3, S4D1 and S1E5, respectively (all $\operatorname{IgM}$ ). A cell line secreting antibody of the fourth category which recognized spores only was not successfully cloned. 

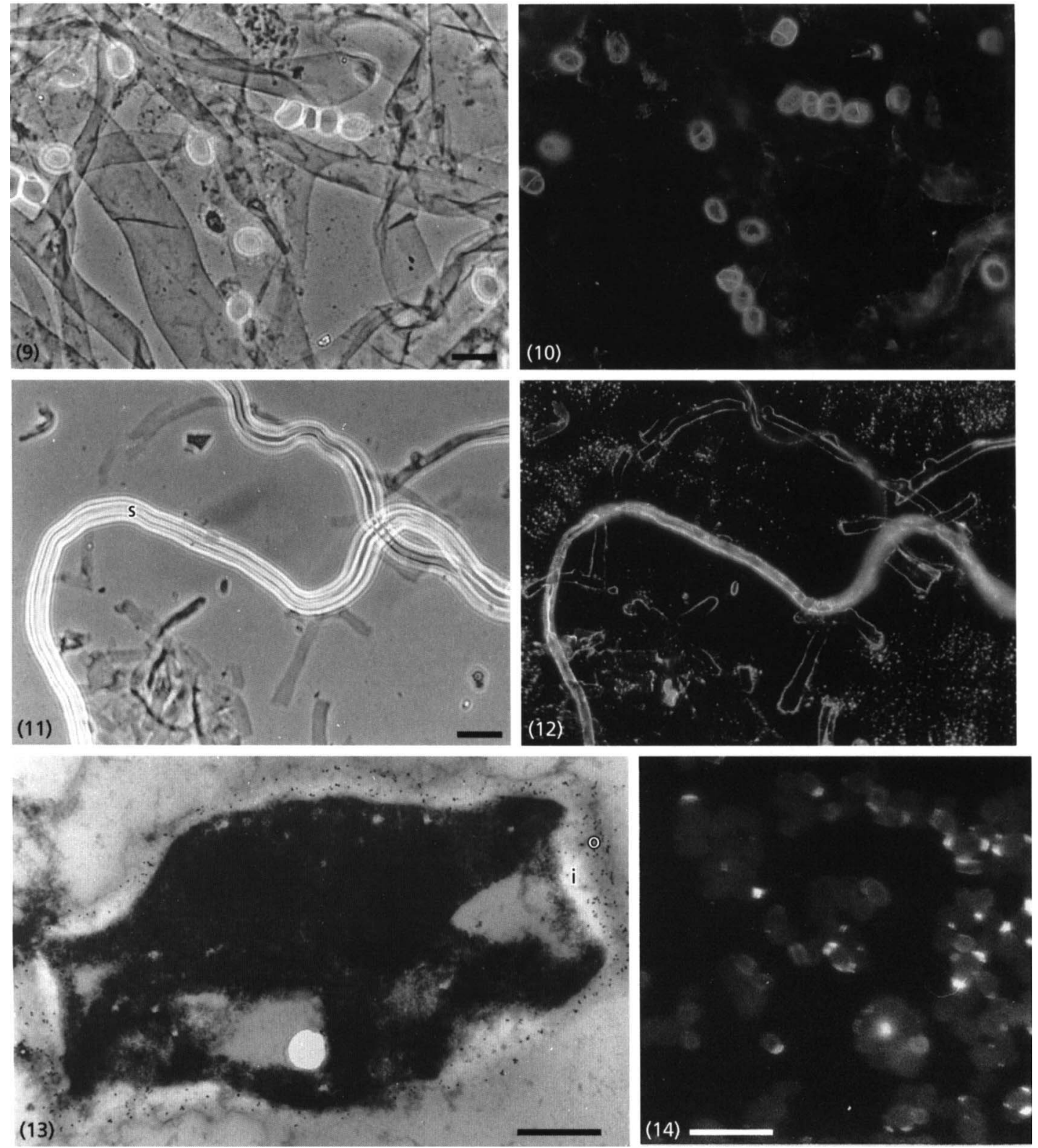

Figs 9-14. Fig. 9. PC micrograph of spores and hyphae from a basidiome of Amanita muscaria treated with mAb S4D1. Bar, $15 \mu \mathrm{m}$. Fig. 10. IF image corresponding to Fig. 9 showing localization of epitope on basidiospores but not on hyphae. Fig. 11. PC micrograph of hyphae from a mycelial culture of Schiz. commune treated with mAb S4D1. A thickwalled skeletal hypha (s) is visible. Bar, $15 \mu \mathrm{m}$. Fig. 12. IF image corresponding to Fig. 11 showing localization of epitope on hyphal surface. Fig. 13. Electron micrograph of longitudinal section through a conidium of $N$. crassa treated with gold-labelled mAb S4D1 showing location of the epitope within both the outer (o) and inner (i) wall layers of the conidium. Bar, $0.5 \mu \mathrm{m}$. Fig. 14. IF micrograph of ungerminated conidia of $N$. crassa treated with mAb S3B3 showing localized distribution of the epitope at some ends of the conidia. Bar, $15 \mu \mathrm{m}$.

From 1440 hybridomas produced using hyphae of $\mathrm{Pax}$. involutus as immunogen, 108 proved positive by ELISA, and 29 of these were also positive in the IF assay. There was only one pattern of reactivity as described below. A representative was cloned and the cell line designated Pax-1, and the antibody it produced, also an IgM, cross-reacted with all fungi against which it was tested (Table 1).

\section{mAb S4D1}

S4D1 recognized an epitope which was distributed over the entire surface of both the dormant conidia (Fig. 1) and hyphae (Figs 2-4); the same immunolabelling pattern was seen with the immunogen $N$. crassa IMI 142819 , and another isolate of N. crassa, IMI 53240, and with other members of the genus (Table 1). S4D1 
reacted to a more limited extent with Sor. fimicola, and showed no reaction with the hyphae of Pod. paucieta, nor with Chaetomium globosum, or arthroconidia of Geotrichum candidum. If $N$. crassa germlings and G. candidum arthroconidia were mixed together and screened for reaction with S4D1 by IF, the mAb clearly differentiated between the two species (Figs 3 and 4).

The reaction of S4D1 with genera outside the Ascomycota was also studied (Table 1). With Pen. chrysogenum, fluorescence was exhibited along the surface of hyphae and conidiophores, but not on the phialides or conidia (Figs 5 and 6). With Aspergillus nidulans, the fluorescence was seen on the conidial surface but it varied in intensity with different conidia (not illustrated). Bright fluorescence was seen on the hyphae, as with Pen. chrysogenum. In the case of Col. gloeosporioides, fluorescence was not seen on the surface of the conidium, appressorium or its subtending primary germ tube, but did occur on the surface of germ tubes ('secondary germ tubes') which were produced after an appressorium had developed (Figs 7 and 8). S4D1 showed no reaction with the hyphae from the fruiting body of Amanita muscaria but fluorescence was observed on the basidiospore surface (Figs 9 and 10). With other members of the Basidiomycota, S4D1 labelled an epitope on the hyphal surface, e.g. with Schiz. commune (Figs 11 and 12) fluorescence was seen particularly along the long unbranched skeletal hyphae, which have thick walls, along with some fluorescence on fragments of thin-walled generative hyphae. It is possible that some of this could be due to autofluorescence.

Thin sections of conidia from a 3-d-old colony of $N$. crassa had a distinct, thin outer wall layer and a thicker, lightly staining inner wall. S4D1 recognized an epitope present throughout the wall layers of the conidium (Fig. 13). The gold labelling was associated with the wall rather than the plasma membrane. The epitope occurred throughout the septal region and was also present between the conidia in chains. The hyphal wall was much thinner than the conidial wall and labelling occurred throughout, although it was not as heavily labelled as the conidial wall (results not illustrated).

Fractions I, II, III and IV from the hyphal walls of $N$. crassa were tested for their reaction with S4D1 by ELISA. S4D1 showed a negligible reaction with Fraction II (monosaccharides), but reacted with the other three fractions (Table 2). It did not react with purified chitin nor with its subunit $\mathrm{N}$-acetylglucosamine. The reaction of S4D1 with Fraction IV (mainly chitin) was not, therefore, due to a reaction with chitin but to a reaction with another hyphal wall component that was also extracted in Fraction IV. Fraction III (glucans) reacted strongly with S4D1, suggesting that the antigen was a major part of this wall fraction. S4D1 also cross-reacted with Fraction I which contained glucan, galactosamine and peptides. The antigen may, therefore, be any one of these, and Fraction III may have contained one of these components as well as the glucan. S4D1 had a negligible reaction with galactosamine, a component of Fraction I
Table 2. $A_{410}$ values for the ELISA reaction of mAb S4D1 with hyphal wall fractions from $N$. crassa and with hyphal wall components prepared from other fungi

Results are expressed as a mean of four replicates (and range of values). Figures quoted have been corrected by subtraction of readings obtained for a second antibody control.

\begin{tabular}{|lc|}
\hline Component & $A_{410}$ \\
\hline Whole N. crassa walls & $1 \cdot 10(1 \cdot 02-1 \cdot 20)$ \\
Fraction I & $0 \cdot 83(0 \cdot 81-0 \cdot 89)$ \\
Fraction II & $0 \cdot 12(0 \cdot 10-0 \cdot 13)$ \\
Fraction III & $1 \cdot 31(1 \cdot 27-1 \cdot 35)$ \\
Fraction IV & $0 \cdot 57(0 \cdot 55-0 \cdot 59)$ \\
A. nidulans $1,3-\alpha$-glucan & $0 \cdot 88(0 \cdot 87-0 \cdot 90)$ \\
Schiz. commune -glucan $(1,3-\alpha)$ & $0 \cdot 70(0 \cdot 65-0 \cdot 79)$ \\
Schiz. commune R-glucan $(1,3-\beta, 1,6-\beta)$ & $0 \cdot 24(0 \cdot 21-0 \cdot 26)$ \\
Chitin & $0 \cdot 00$ \\
$N$-Acetylglucosamine & $0 \cdot 00$ \\
Galactosamine & $0 \cdot 00$ \\
\hline
\end{tabular}

but reacted with a 1,3- $\alpha$-glucan isolated from hyphal walls of $A$. nidulans and a 1,3- $\alpha$-glucan (or S-glucan) isolated from hyphal walls of Schiz. commune (Table 2). In contrast, it reacted only very weakly with $1,3-\beta-, 1,6-$ $\beta$-glucan (or R-glucan) from hyphal walls of Schiz. commune (Table 2).

\section{mAb S3B3}

S3B3 recognized an epitope present at the ends of some conidia (Figs 14, 16 and 18). The epitope, however, was not always present at both or even one end of all detached conidia (Fig. 14). In a conidial chain, the fluorescence occurred in the septal region (Figs 15-18). Conidial chains of $N$. crassa develop basipetally, and the position of a conidium in the chain may correlate with the presence or absence of the epitope. For example, in Figs 17 and 18 in the older portion of the conidiophore, the septal region showed fluorescence, whereas in the developing conidial chains there was no fluorescence until the septal crosswalls formed.

S3B3 cross-reacted in a similar fashion with the other Neurospora isolates but showed no reaction with Sor. fimicola or Pod. paucieta (Table 1). It did not react with the ends of the arthroconidia of G. candidum, which are also formed basipetally, or with conidia from $A$. nidulans, but reacted weakly with the ends of conidia from Pen. chrysogenum. No reaction was seen with any member of the Basidiomycota or Zygomycota tested.

Immunolabelling at the electron-microscopic level also showed the epitope recognized by S3B3 to be localized at the ends of conidia, as might be expected from the IF pattern shown by light microscopy, but the label was also seen more generally within the inner walls of conidia (Figs 19, 20), as well as within the hyphal walls 

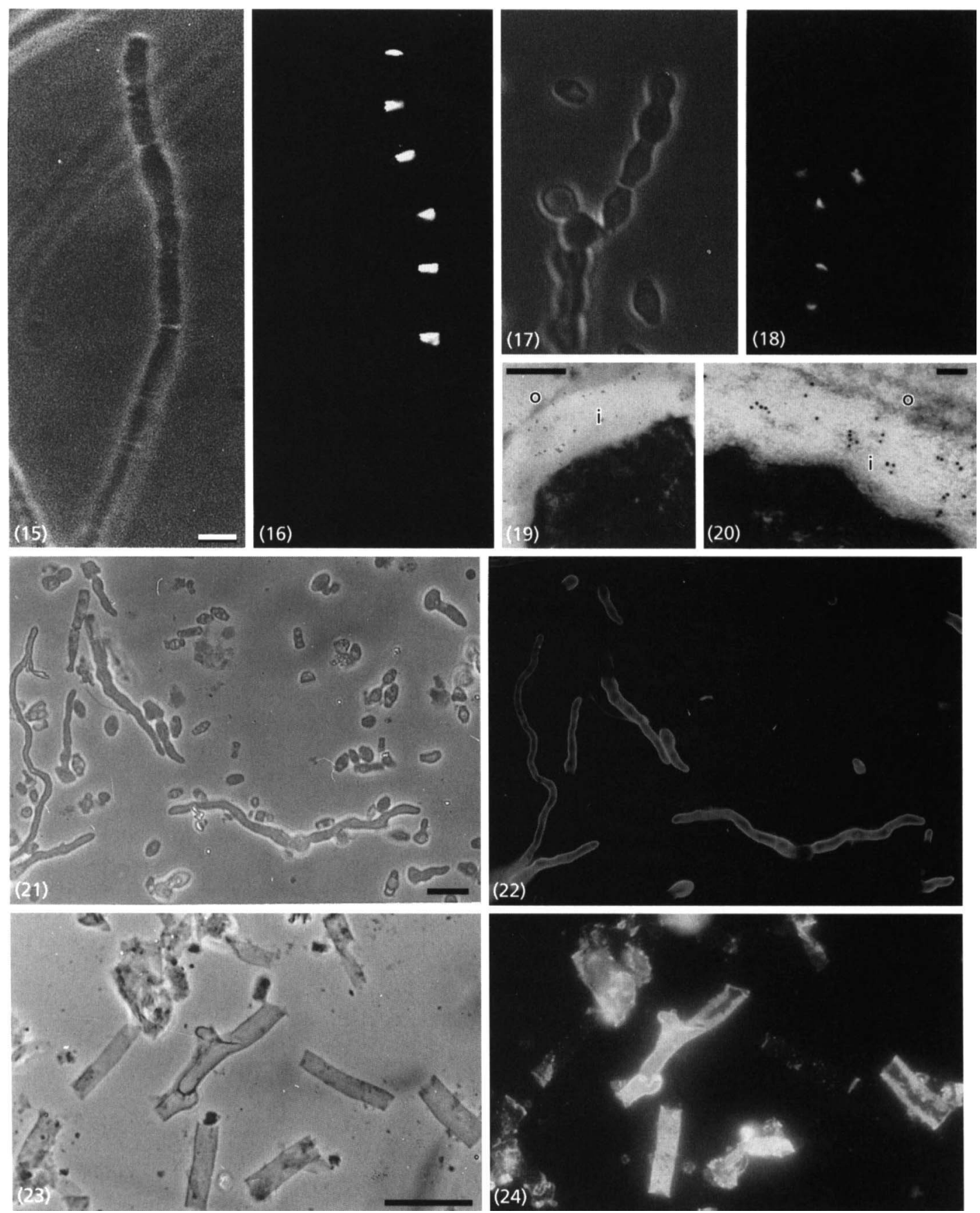

Figs 15-24. Figs 15 and 17. PC micrographs of developing conidial chains of $N$. crassa treated with mAb S3B3. Bar, $5 \mu \mathrm{m}$. Figs 16 and 18. IF images corresponding to Figs 15 and 17 respectively showing localized distribution of epitope only between the ends of mature conidia. Figs 19 and 20. Electron micrographs of longitudinal sections through conidial wall of $N$. crassa treated with gold-labelled mAb S3B3 showing location of the epitope within the inner electronlucent wall layer (i) and not in the outer wall (o). Bar, $0.5 \mu \mathrm{m}$ (Fig. 19), $0.1 \mu \mathrm{m}$ (Fig. 20). Fig. 21. PC micrograph of germlings and ungerminated conidia of $N$. crassa treated with mAb S1E5. Bar, $15 \mu \mathrm{m}$. Fig. 22. IF image corresponding to Fig. 21 showing localized distribution of the epitope over the surface of germ tubes but not on the conidial surface. Fig. 23. PC micrograph of hyphae from a mycelial culture of Pax. involutus treated with mAb Pax-1. Bar, 15 $\mu \mathrm{m}$. Fig. 24. IF image corresponding to Fig. 23 showing localization of epitope over hyphal surface.

and in the septal region of hyphae. In sections of conidial chains, gold labelling was also seen in the septal region (not illustrated). Because the binding pattern of this
$\mathrm{mAb}$ resembled that of Calcofluor, which binds to chitin and other $\beta$-linked glucans, the antibody was also screened by ELISA against pure chitin and $\mathrm{N}$-acetyl- 
glucosamine (as described for S4D1) but was found not to bind to either.

\section{mAb S1E5}

SIE5 reacted with an epitope present on the surface of germ tubes of $N$. crassa, but which was not present on dormant conidia (Figs 21, 22). Labelling of conidia was occasionally observed but these were usually swollen conidia in the process of germination. The reaction with heterologous fungi was not tested. Although the fluorescence pattern exhibited by conidia showed that the epitope was not present at the surface of conidia, immunogold labelling of thin sections of conidia revealed that the epitope was present in the inner wall layers, but was not present at the conidial surface (results not shown).

\section{Pax-1}

Pax-1 reacted with hyphal walls of Pax. involutus ITE 16 (Figs 23, 24). It also reacted with hyphal walls from Pax. involutus ITE32, and hyphae and basidiospores from a fresh basidiome of Pax. involutus. In a population of hyphal fragments, some showed fluorescence along their whole length, some show fluorescence along only part of the fragment and others showed no fluorescence at all. The strongest fluorescence seen was often associated with the ends of the fragments and this suggested that Pax-1 reacted with an internal antigen. Pax-1 possibly reacted with an epitope distributed at the surface only at certain times during hyphal development. The pattern seen on older mycelial strands did not differ significantly from that on younger wall fragments.

Pax -1 reacted by IF with hyphae and spores of 22 species of Basidomycota tested (including species of Agaricus, Amanita, Boletus, Clitopilus, Collybia, Coprinus, Gymnopilus, Hebeloma, Laccaria, Lactarius, Mycena, Pluteus and Russula); the pattern of fluorescence was similar to that for Pax. involutus (data not shown). Pax1 did not react with $M$ ucor biemalis but cross-reacted, however, with members of the Ascomycota (Table 1). Pax-1 showed the same pattern of fluorescence on conidia of $N$. crassa as S3B3. The different patterns of reactivity against other antigens, however, suggest that these two mAbs are not identical.

\section{DISCUSSION}

The four types of IF reaction reported here indicate the potential of $\mathrm{mAbs}$ in the localization of particular wall components. $\mathrm{mAb}$ S4D1, for example, recognized an antigen present on the surface of both conidia and hyphae in N. crassa, and in the glucan fraction of hyphal walls of $N$. crassa, and the 1,3- $\alpha$-glucan fractions from A. nidulans and Schiz. commune. The presence of $1,3-\alpha-$ glucans has been reported from the hyphal walls of N. crassa (Bull, 1970; Cardimil \& Pincheira, 1979), and our results suggest that this component is distributed throughout the walls of both hyphae and conidia and not localized in a specific region. mAb S4D1 was also used to localize the epitope in other fungi. All fungi tested from the Ascomycota and Basidiomycota reacted with the antibody, whereas fungi from the Zygomycota did not. This provides further circumstantial evidence that $1,3-\alpha-$ glucan is not present in the hyphal walls of Zygomycota (Bartnicki-Garcia, 1968). mAbs have previously been found which react to epitopes within particular carbohydrate components of the hyphal wall, but these have usually been to $\beta$-linked glucans, the most important group of fungal glucans (Ruiz-Herrera, 1991).

The surface distribution of the S4D1 epitope varies between fungal species, and also within different structures of a single species. The differential expression and location of epitopes in different structures of the same fungus at varying development stages has been observed previously with other fungi (e.g. Hardham et al., 1985, 1994; Wycoff \& Ayers, 1990; Ollert \& Calderone, 1990; Xia et al., 1993). A comparable result was observed during IF and immunogold labelling of mycelium of Penicillium islandicum with a mAb raised to soluble surface molecules (Cole et al., 1991). The antibody bound to outer wall layers in actively growing young hyphae and to regions within the wall of older mycelium. It did not bind to phialides or conidia. Conversely, an antibody raised to phialoconidia of Trichoderma harzianum reacted with the immunogen but not with the mycelium or chlamydospores of the same fungus (Thornton \& Dewey, 1996). mAb S4D1 bound to hyphae but not to appressoria of Col. gloeosporioides. These latter structures are heavily melanized (Jeffries et al., 1990) which may mask the relevant epitope. mAbs raised against Colletotrichum lindemuthianum have previously been shown to exhibit differential binding patterns (Pain et al., 1992, 1994b). One antibody (UB20) labelled conidia strongly, but germ tubes and appressoria weakly; another (UB22) reacted strongly with germ tubes, especially in regions away from the apices, as well as labelling appressoria and conidia less strongly; a third antibody (UB25) bound only to intracellular hyphae and did not recognize conidia, appressoria or germ tubes.

Although a large number of hybridomas were raised which produced $\mathrm{mAbs}$ that gave positive results in both ELISA and IF screens against hyphal walls, there was a relative lack of variation in the IF patterns obtained. This suggests immunodominance by some wall components or a uniformity in hyphal wall composition. Immunodominance has been reported in previous studies. In Aspergillus and Penicillium species, for example, some extracellular polysaccharides are immunodominant (Notermans et al., 1987, 1988) and the use of crude mycelial extracts as immunogens has been shown to induce cross-reactive antibodies. Many of the cell lines we obtained gave IF patterns similar to mAb S3B3, whereas the other binding patterns were only demonstrable with a few cell lines. Thus, immunodominance was most strongly exhibited by the material which accumulates between conidia during sporogenesis. The 
morphological development of conidiophores of $N$. crassa involves the septation of a preconidial stage such that individual conidia become delimited by crosswalls (Springer \& Yanofsky, 1989). When the interconidial junction has reached the furrow stage, IF staining of this region using Calcofluor reveals two thick bands of material similar to those obtained when reacted with $\mathrm{mAb}$ S3B3. Calcofluor binds to chitin and other $\beta$-linked glucans whereas $\mathrm{mAb} S 3 \mathrm{~B} 3$ does not react to chitin or to $\mathrm{N}$-acetylglucosamine, the chitin subunit, thus it is evident that these two fluorescence probes are reacting to different molecules which have a similar distribution.

In our work, the use of crude mycelial homogenates has revealed a degree of diversity of responses to unrefined immunogens. The individual reactions of mAbs S4D1, S3B3, S1E5 and Pax-1 to hyphae of N. crassa show differential surface localization of their respective epitopes. Previous tests using ELISA with homologous antigens have also shown that different $m A b s$ raised against the same immunogens can react differently with different fungal structures (Xia et al., 1992). Immunodominant antigens, however, can mask these more specific interactions and this emphasizes the need to screen a large number of cell lines to obtain a full spectrum of responses. By this means it is possible to get a range of mAbs that are excellent probes for small regions of hyphal wall morphogenesis and these should be very useful in determining the structural complexity of hyphal development.

\section{ACKNOWLEDGEMENTS}

The senior author thanks the NERC for the award of a studentship. A. nidulans $1,3-\alpha$-glucan and the glucans from Schiz. commune were gifts to Professor K. Gull from Professor B. J. M. Zonneveld and Professor J. G. H. Wessels, respectively. We thank Dr P. A. Mason of the ITE for cultures of some of the Basidiomycota, and Ray Newsam for technical assistance.

\section{REFERENCES}

Banks, J. N. \& Cox, S. J. (1992). The solid phase attachment of fungal hyphae in an ELISA to screen for antifungal antibodies. Mycopathologia 120, 79-85.

Bartnicki-Garcia, S. (1968). Cell wall chemistry, morphogenesis and taxonomy of fungi. Annu Rev Microbiol 22, 87-108.

Bendayan, M. (1984). Enzyme-gold electron microscopic cytochemistry: a new affinity approach for the ultrastructural localization of macromolecules. J Electron Microsc Techn 1, 349-372.

Benhamou, N., Chamberland, H. \& Noel, S. (1990). Ultrastructural localization of $\beta$-1,4-glucan-containing molecules in the cell walls of some fungi : a comparative study between spore and mycelium. Can J Microbiol 36, 149-158.

Breuil, C., Luck, B. T., Rossignol, L., Little, J., Echeverri, J., Banerjee, S. \& Brown, D. L. (1992). Monoclonal antibodies to Gliocladium roseum, a potential biological control fungus of sapstaining fungi in wood. J Gen Microbiol 138, 2311-2319.

Bull, A. T. (1970). Chemical composition of wild-type and mutant Aspergillus nidulans cell walls. The nature of polysaccharide and melanin constituents. J Gen Microbiol 63, 74-94.
Cardimil, L. \& Pincheira, G. (1979). Characterization of the carbohydrate component of Fraction I in the Neurospora crassa cell wall. J Bacteriol 137, 1067-1072.

Chang, P. K. Y. \& Trevithick, J. P. (1972). Release of wall-bound invertase and trehalose in Neurospora crassa by hydrolytic enzymes. J Gen Microbiol 70, 13-22.

Cole, L., Hawes, C. \& Dewey, F. M. (1991). Immunocytochemical localization of a specific antigen in cell walls of Penicillium islandicum. Mycol Res 95, 1369-1374.

Gianinazzi-Pearson, V., Lemoine, M.-C., Arnould, C., Gollote, A. \& Morton, J. B. (1994). Localization of $\beta(1 \rightarrow 3)$ glucans in spore and hyphal walls of fungi in the Glomales. Mycologia 86, 478-485.

Hardham, A. R., Susaki, E. \& Perkin, J. L. (1985). The detection of monoclonal antibodies for specific components on the surface of zoospores and cysts of the fungus Phytophthora cinnamomi. Exp Mycol 9, 264-268.

Hardham, A. R., Cahill, D. M., Cope, M., Gabor, B. K., Gubler, F. \& Hyde, G. J. (1994). Cell surface antigens of Phytophthora spores: biological and taxonomic characterization. Protoplasma 181, 213-232.

Hunsley, D. \& Burnett, J. H. (1970). The ultrastructural architecture of walls of some hyphal fungi. J Gen Microbiol 62, 203-218.

Hunsley, D \& Kay, D. (1976). Wall structure of the Neurospora hyphal apex: immunofluorescent localization of wall surface antigens. J Gen Microbiol 95, 233-248.

Jeffries, P., Dodd, J. C., Jeger, M. J. \& Plumbley, R. A. (1990). The biology and control of Colletotrichum species on tropical fruit crops. Plant Pathol 39, 343-366.

Mahadevan, P. R. \& Tatum, E. L. (1965). Relationship of the major constituents of the Neurospora crassa cell wall to wild-type and colonial morphology. J Bacteriol 90, 1073-1081.

Marshall, M. (1990). Immunological recognition of fungi. $\mathrm{PhD}$ thesis, University of Kent.

Marx, D. H. (1969). The influence of ectotropic mycorrhizal fungi on the resistance of pine roots to pathogenic infections. I. Antagonism of mycorrhizal fungi to root pathogenic fungi and soil bacteria. Phytopathology 59, 153-163.

Miller, S. A. \& Martin, R. R. (1988). Molecular diagnosis of plant disease. Annu Rev Phytopathol 26, 409-432.

Notermans, S., Wieten, G., Engel, H. W. B., Rombouts, F. M., Hoogerhout, P. \& Van Boom, J. H. (1987). Purification and properties of extracellular polysaccharide (EPS) antigens produced by different mould species. J Appl Bacteriol 62, 157-166.

Notermans, S., Veeneman, G. H., Van Zuylen, C. W. E. M., Hoogerhout, P. \& Van Boom, J. H. (1988). (1 $\rightarrow 5)$-linked $\beta$-D-galactofuranosides are immunodominant in extracellular polysaccharides of Penicillium and Aspergillus species. Mol Immunol 25, 975-979.

Ollert, M. W. \& Calderone, R. A. (1990). A monoclonal antibody that defines a surface antigen on Candida albicans hyphae crossreacts with yeast cell protoplasts. Infect Immun 58, 625-631.

Pain, N. A., O'Connell, R. J., Bailey, J. A. \& Green, J. R. (1992). Monoclonal antibodies which show restricted binding to four Colletotrichum species: C. lindemuthianum, C. malvarum, C. orbiculare and C. trifolii. Physiol Mol Plant Patbol 41, 111-126.

Pain, N. A., Green, J. R., Gammie, F. \& O'Connell, R. J. (1994a). Immunomagnetic isolation of viable intracellular hyphae of Colletotrichum lindemuthianum (Sacc. \& Magn.) Briosi \& Cav. from infected bean leaves using a monoclonal antibody. New Phytol 127, 223-232. 
Pain, N. A., O'Connell, R. J., Mendgen, K. \& Green, J. R. (1994b). Identification of glycoproteins specific to biotrophic intracellular hyphae formed in Colletotrichum lindemuthianum-bean interaction. New Phytol 127, 233-242.

Ruiz-Herrera, J. (1991). Biosynthesis of $\beta$-glucans in fungi. Antonie Leeuwenhoek 60, 73-81.

Schots, A., Dewey, F. M. \& Oliver, R. (1994). Modern Assays for Plant Pathogenic Fungi: Identification, Detection and Quantification. Wallingford: CAB International.

Springer, M. L. \& Yanofsky, C. (1989). A morphological and genetic analysis of conidiospore development in Neurospora crassa. Genes Dev 3, 559-571.

Ste-Marie, L., Senechal, S., Bouschira, M., Garzon, S., Strykowski, H., Pedneault, L. \& Repentigny, L. D. (1990). Production and characterization of monoclonal antibodies to cell wall antigens of Aspergillus fumigatus. Infect Immun 58, 2105-2114.

Thornton, C. R. \& Dewey, F. M. (1996). Detection of phialoconidia of Trichoderma harzianum in peat-bran by monoclonal antibody- based enzyme-linked immunosorbent assay. Mycol Res 100, 217-222.

Thornton, C. R., Dewey, F. M. \& Gilligan, C. A. (1993). Development of monoclonal antibody-based immunological assays for the detection of live propagules of Rhizoctonia solani in soil. Plant Pathol 42, 763-773.

Wycoff, K. L. \& Ayers, A. R. (1990). Monoclonal antibodies to surface and extracellular antigens of a fungal plant pathogen, Phytophthora megasperma f.sp. glycinea, recognise specific carbohydrate epitopes. Physiol Mol Plant Pathol 37, 55-79.

Xia, J. Q., Lee, F. N., Scott, H. A. \& Raymond, L. R. (1992). Development of monoclonal antibodies specific for Pyricularia grisea, the rice blast pathogen. Mycol Res 96, 867-873.

Xia, J. Q., Lee, F. N. \& Spiegel, F. W. (1993). Characterization of monoclonal antibodies to conidial antigens of Pyricularia grisea. Exp Mycol 17, 170-181.

Received 31 January 1997; accepted 18 March 1997. 